\title{
IRE1 RNase controls CD95-mediated cell death
}

Pelizzari-Raymundo $D^{1,2}$, Le Gallo $M^{1,2}$, Pineau $R^{1,2}$, Papaioannou $A^{1,2}$, Martin $S^{1,2}$, Avril $\mathrm{T}^{1,2}$, Chevet $\mathrm{E}^{1,2}$, Lafont $\mathrm{E}^{1,2^{*}}$

${ }^{1}$ Inserm U1242, University of Rennes, Rennes, France.

${ }^{2}$ Centre de Lutte Contre le Cancer Eugène Marquis, Rennes, France.

${ }^{*}$ Corresponding author: elodie.lafont@inserm.fr

Keywords: CD95/Cell death/ER stress/Unfolded Protein Response/IRE1

Running title: IRE1 RNase controls CD95-mediated cell death 


\begin{abstract}
Unfolded Protein Response (UPR) and Death Receptor (DR) signalling are cellular stress pathways frequently activated towards pro-tumoral cellular outputs in cancer. Experimental evidence has highlighted functional links between the UPR and signalling by the DR TRAIL-R1/2. Herein, we demonstrate that the UPR sensor IRE1 controls the expression of the DR CD95/Fas, and its cell death-inducing capacity. Whereas CD95 is not a general determinant of ER stress-induced cell death, IRE1 RNase activity inhibition increased CD95 expression and exacerbated CD95L-induced cell death in glioblastoma (GB) and Triple-Negative Breast Cancer (TNBC) cell lines. In accordance, CD95 mRNA was identified as a target of the Regulated IRE1-Dependent Decay of RNA (RIDD). Moreover, CD95 expression is elevated in TNBC and GB human tumours exhibiting low RIDD activity. Surprisingly, CD95 expression is also reduced in XBP1s-low human tumour samples. We show that IRE1 RNase inhibition led to CD95 expression attenuation and reduced CD95-mediated hepatic toxicity in mice. In addition, overexpression of XBP1s sensitized GB and TNBC cells to CD95Linduced cell death. Overall, these results demonstrate the tight IRE1-mediated control of CD95-dependent cell death signals in a dual manner through both RIDD and XBP1s, and they identify a pharmacologically actionable link between IRE1 and CD95 signalling.
\end{abstract}




\section{Introduction}

Throughout tumour development, cancer cells are subjected to both intrinsic and extrinsic stresses to which they must adapt to survive and proliferate. Adapted tumour cells survive this selection pressure through the activation of specific signalling pathways. Amongst those are the signalling engaged by the Death Receptors (DR) and the Unfolded Protein Response (UPR), an adaptive response to Endoplasmic Reticulum (ER) stress. The DR TRAIL-R1/2 and CD95 contribute to the immunosurveillance towards cancer or infected cells by their ability to induce cell death upon engagement by their ligand, TRAIL and CD95L, respectively ${ }^{1-3}$. In some tumour cells however, engagement of CD95 or TRAIL-R1/2 fails to induce cell death and promotes pro-tumorigenic cellular outcomes. Similarly, constitutive UPR activation is often observed in cancer cells, resulting from adaptation to various stresses including oncogenic insults, aneuploidy or nutrient deprivation. This constitutive activation does not result in cell death, as would be the case in normal cells, but rather allows cancer cells to thrive through activation of pro-tumoral outcomes ${ }^{4,5}$. Thus, both UPR and DR signalling contribute to tumour progression and relapse in various cancer models including glioblastoma (GB) ${ }^{6-11}$ and Triple Negative Breast Cancer (TNBC) ${ }^{12-16}$. The UPR consists of three core signalling branches initiated by the activation of three sensors upon ER stress. These sensors are ATF6 $\alpha$ (for Activating Transcription Factor 6 alpha), IRE1 $\alpha$ (for Inositol-Requiring Enzyme 1 alpha, referred to as IRE1 hereafter) and PERK (for Protein kinase R-like ER Kinase). IRE1 RNase activation upon ER stress drives (i) the unconventional splicing of XBP1 mRNA, ultimately leading to the expression of the transcription factor XBP1s, which in turn promotes the expression of multiple genes aimed at restoring ER homeostasis and (ii) the degradation of RNA through Regulated IRE1-Dependent Decay of RNA (RIDD). RIDD leads to both cytotoxic and non-cytotoxic cellular outcomes ${ }^{17}$. In addition to their individual roles in controlling cell fate, both UPR and DR signalling are functionally intertwined ${ }^{18,19}$. Indeed, TRAIL-R2, and in some cases TRAIL-R1 and TRAIL, are upregulated downstream of the PERK/ATF4 and/or CHOP axis, in various ER stress conditions and accordingly TRAIL-R signalling can participate to cell death upon ER stress ${ }^{18-28}$. On the contrary, RIDD limits TRAIL-R2-induced signalling by reducing the abundance of its mRNA ${ }^{22}$. Moreover, TRAIL-R2 was recently shown to be directly activated intracellularly by misfolded proteins and therefore signals apoptosis from intracellular compartments $^{29}$. Although the relationships between ER stress and TRAIL-R have been relatively well explored, whether and how IRE1 and CD95 signalling are also linked remains however, unclear. We have recently identified CD95 mRNA as being cleaved by IRE1 RNase in an in vitro RNA cleavage assay ${ }^{11}$. Herein, we investigate the impact of IRE1 RNase activity in CD95 signalling, revealing a previously unrecognised dual functional link between these pathways.

\section{Results}

\section{Basal and ER-stress induced IRE1 activation limits CD95 expression in GB and TNBC cells}

We have recently identified CD95 mRNA as being cleaved by IRE1 in an in vitro RNA cleavage assay ${ }^{11}$. To evaluate if the level of CD95 mRNA is controlled by IRE1 in a cellular context, we used the U87 GB cell line, which displays a constitutive activation of IRE1 ${ }^{11}$. Expression of a dominant-negative (DN) form of IRE1, which represses the activation of endogenous IRE1, and thus its RNase activity ${ }^{30,31}$, led to a significant increase in the basal level of CD95 mRNA (Fig 1A). Accordingly, cell surface expression of CD95 was heightened in IRE1 DN-overexpressing U87 cells as 
compared to WT cells (Fig 1B). Similar results were obtained in two primary GB cell lines, RADH87 and RADH85 ${ }^{32}$ in which expression of IRE1Q780*, a mutant of IRE1 devoid of both kinase and RNase domains that blunts IRE1 signalling, led to an increased expression of CD95 mRNA (Fig 1A) as well as of total and cell surface (Fig S1A and Fig 1B) CD95 protein. To determine if exogenous activation of IRE1 impacts on CD95 expression, we next analysed CD95 expression levels in U87 cells and in the TNBC cell line SUM159 treated or not with ER stress inducers. In this context, both tunicamycin (TM) and MG-132 promoted CD95 mRNA degradation, which was prevented by treatment with MKC-8866, a pharmacological inhibitor of IRE1 RNase activity ${ }^{33}$ (Fig S1B and C). A consistent result was obtained regarding CD95 protein expression. Indeed, ER stress induced by TM or thapsigargin (TG) provoked a decrease in CD95 protein level (Fig 1C-F) which was reverted by MKC-8866. Overall, these results highlight that both constitutive and ER stress-induced IRE1 RNase activities limit CD95 expression in the tested cellular models.

\section{IRE1 cleaves CD95 mRNA in vitro}

To test whether CD95 mRNA is a target of the RIDD, we first evaluated the ability of recombinant IRE1 to cleave CD95 mRNA in vitro. This revealed that CD95 mRNA is indeed directly targeted by IRE1 RNase activity (Fig 2A). We then searched the sequence of CD95 mRNA for the presence of potential RIDD cleavage sites. Such sites have been reported to both (i) reside in hairpin loop structures and (ii) display a consensus sequence CNG/CAGN ${ }^{34-36}$. However, no such site was identified in the CD95 mRNA sequence. We, therefore, expanded our search to additional potential sequences beyond this classical consensus. These supplementary sequences (CAACAA, CAGCUC, CUGCAU and CUGGCG) can be targeted by IRE1 in vitro when displayed on hairpin loops as recently identified in our laboratory ${ }^{37}$. Based on this second analysis, we identified two potential cleavage sites, one located within the CD95 ORF and the other in the 3'UTR of CD95 mRNA (Fig 2B). Noteworthy, amplification of $136 \mathrm{bp}$ and $121 \mathrm{bp}$ regions encompassing the CAACAA and CUGCAU sites respectively was dampened when performed from RNA incubated with recombinant IRE1, arguing that these two sites might indeed be targeted by IRE1 in vitro (Fig 2C, D). Taken together, these results indicate that CD95 mRNA is a bona fide substrate of IRE1 RNase in vitro.

\section{CD95 is not a general determinant of ER stress-induced cell death}

Since the expression of another DR, TRAIL-R2, is also regulated by IRE1 ${ }^{22}$ and that TRAIL-R2 signalling impacts on ER stress-induced cell death, we next hypothesized that CD95 may also influence the sensitivity of cells to ER stress. To test this hypothesis, we used U87 cells in which CD95 was knocked-down using RNA interference as well as MDA-MB-231 TNBC cells WT or KO for CD95 (Fig S2A, B). Whereas CD95 depletion led to a reduced sensitivity to TM-induced loss of viability in both cell lines, it did not impact any of these cells' ability to die in response to TG, MG132 and Brefeldin A (Fig 3A, B and Fig S2A, B). These results, therefore, imply that while CD95 might contribute to cell death in response to some specific ER homeostasis insults or to aberrant protein N-linked glycosylation, this DR is not a universal determinant of cells' sensitivity to ER stress-induced cytotoxicity.

IRE1 RNase activity limits CD95L-induced caspase-activation and cell death Since our cell-based results indicate that the RIDD limits CD95 expression, we hypothesized that IRE1-mediated depletion of CD95 represses CD95L-induced cell 
death. Indeed, U87 expressing IRE1 DN cells were markedly sensitized to CD95Linduced loss of viability (Fig 3C). In line with this observation, activation of the initiator caspase-8 and the effector caspase-3 happened earlier and was more pronounced in IRE1 DN-overexpressing U87 cells as compared to WT cells (Fig 3D), further pointing out that IRE1 controls an early event in CD95 cell death signalling. Noteworthy, RADH85 cells expressing IRE1Q780* were also sensitized to CD95L-induced cell death (Fig 3E). Yet, despite increased expression of CD95 (Fig 1B), RADH87 expressing IRE1Q780* were not sensitized to CD95L-induced cell death (Fig 3F), leading us to postulate that an additional cell death-inhibitory checkpoint, downstream of CD95, might be active in these cells. To test this hypothesis, we targeted the antiapoptotic proteins cIAP1/2 and XIAP which are known negative regulators of CD95mediated cell death ${ }^{38,39}$. To this end, we utilized the SMAC mimetic Birinapant which potently inhibits cIAP1 and, albeit to a lesser extent, cIAP2 and XIAP ${ }^{40,41}$. RADH87 expressing IRE1Q780* were notably sensitized to CD95L-induced loss of viability when co-incubated with Birinapant as compared to control or IRE1 WT-overexpressing cells (Fig 3F). Taken together, these data argue for an early role of IRE1 in limiting CD95Linduced cell death signalling.

\section{Impact of IRE1 RNase inhibition in CD95-mediated cell death in an acute liver injury model}

To determine whether IRE1 RNase activity controls CD95 expression and signalling in vivo, we first evaluated the impact of intra-peritoneal injection of MKC-8866 on CD95 expression in mouse livers (Fig 4A). In contrast with the results previously obtained in cancer cell lines (Fig 1-2), this analysis indicated that the pharmacological inhibition of IRE1 RNase led to decreased CD95 protein expression in vivo (Fig 4 B). To investigate whether this unexpected reduction of CD95 expression levels resulted in a decreased sensitivity to CD95-mediated cell death, we next used the anti-CD95 agonist antibody Jo2. When utilized at a sub-lethal dose, Jo2 induced a mild hepatitis within 6 hours of treatment ${ }^{42}$. Mice pre-treated with MKC-8866 or vehicle were therefore injected with Jo2 or the corresponding isotype control (Fig S3A). First, immunohistochemical (IHC) analysis of the liver sections confirmed that inhibition of IRE1 led to a reduction in CD95 expression (Fig S3B). Furthermore, both HES and IHC analysis of the same liver sections using an anti-cleaved caspase-3 indicated a clear reduction of Jo2-induced liver damage in the MKC-8866-treated group as compared to vehicle-treated animals (Fig 4C and Fig S3C), a result which was further confirmed by Western blot (Fig S3D). Taken together, these results indicate that IRE1 RNase activity promotes CD95 hepatic expression and CD95-mediated hepatotoxicity in vivo.

\section{Dual regulation of CD95 expression and signalling by IRE1 RNase activity}

To further investigate the apparent discrepancy in the results obtained in cell lines and in vivo, we explored how the different branches of IRE1 signalling impact on CD95 expression. Indeed, IRE1 RNase activity can mediate both the RIDD and the unconventional splicing of XBP1 mRNA, the latter resulting in the expression of the XBP1s transcription factor. Since we observed that MKC-8866 dampened CD95 expression and CD95-mediated cell death in vivo, we thus hypothesized that XBP1s, contrary to the RIDD, may enhance CD95-mediated cell death. This hypothesis was validated as we observed that overexpression of XBP1s in both U87 and SUM159 increased CD95L-induced cell death (Fig 5A, B). So far, CD95 has not been reported as a genuine XBP1s-target gene and it therefore remains to be evaluated whether this transcription factor sensitizes cells directly through up-regulation of this DR, of 
downstream pro-apoptotic factors of this pathway, a combination thereof or additional more indirect mechanisms. Nevertheless, taken together with our in vivo data (Fig 4), our results point towards a role for XBP1s in promoting CD95-mediated cell death. Collectively our results may therefore indicate a dual regulation of CD95 expression and signalling by IRE1 RNase activity. Next, we explored whether the activation of the RIDD and XBP1s branches of IRE1 RNase activity might correlate with CD95 mRNA expression levels in human tumours. Thus, we analysed the expression of this DR mRNA in tumours classified according to their RIDD and XBP1s gene expression signature, as defined previously ${ }^{11}$. This analysis revealed that XBP1s-high tumours present a significantly heightened expression of CD95, as compared to XBP1s-low tumours (Fig 5C). Conversely, RIDD-high tumours present a significantly diminished CD95 mRNA level when compared to RIDD-low tumours (Fig 5D), hence indicating a dual regulation of CD95 mRNA expression by IRE1 signalling.

\section{Discussion}

Both UPR and DR signalling contribute to the elimination of cancer cells whereas diversion of these pathways towards pro-tumorigenic outcomes can promote tumour progression. So far, extensive experimental evidence demonstrated that TRAILR-1/2signalling and the UPR are functionally linked, via PERK and IRE1 through its RIDD activity, to control cell fate ${ }^{18-28,43}$. TRAIL-R1/2-emanating signals not only contribute to ER stress-induced cell death but also to cytokine production ${ }^{44}$. Furthermore, it has recently been shown that TRAIL-R2 can directly bind to and get activated by misfolded proteins ${ }^{29}$ to promote cell death from intracellular compartments ${ }^{45}$. Of note, the TRAILR1 gene has also been identified as an XBP1s target in a chromatin immunoprecipitation experiment, and it is thus tempting to speculate that the expression of this DR might be directly inducible by this transcription factor at least in some cell types ${ }^{12}$. With regards to CD95, it was previously reported that its expression can be induced by ER stress in macrophages, a phenomenon which was attributed to a calcium/calmodulin-dependent protein kinase IIy (CaMKIl $\gamma$ )-dependent pathway ${ }^{46}$. In addition, in INS-1E rat insulinoma cells, it was suggested that cyclopiazonic acid could enhance IL-1-induced CD95 expression in an XBP1s-dependent manner ${ }^{47}$. Therefore, the existence of a potential connection between ER homeostasis control and CD95 in cancer cells remained largely uncharted. Herein, we observe that IRE1 RNase cleaves CD95 mRNA in vitro and IRE1 activity represses CD95 expression (at both mRNA and protein levels) as well as CD95L-induced cell death in TNBC and GB cell lines. Accordingly, CD95 mRNA expression is reduced in both GB and TNBC human tumours displaying an elevated RIDD activity. Unexpectedly, we observed that expression of CD95 mRNA is increased in XBP1s-high tumours. This observation coincided with the fact that overexpression of XBP1s sensitizes cells to CD95Linduced cell death and that pharmacological inhibition of IRE1 RNase represses both CD95 hepatic expression and the hepatotoxicity induced by a CD95-targeting agonistic antibody. Therefore, our study highlights a previously unrecognised and dual link between IRE1 activity and the expression and signalling of CD95 (Fig 5E).

The pathophysiological importance of such connection remains to be explored, but given the increasing variety of conditions that are influenced by CD95 and IRE13, 4 , we deem it likely to be broadly relevant. One such context could be tumour progression. Indeed, the ability of IRE1 to dually regulate CD95-mediated cell death suggests that the preferential branch activated by this RNase in cancer cells could be one of the determinants of their response to endogenous immunosurveillance or even T-cell immunotherapy ${ }^{48,49}$. CD95 can also mediate a variety of non-cytotoxic pro- 
tumoural cellular outcomes, including migration, production of pro-inflammatory cytokines, proliferation and regulation of cell differentiation state, which are also modulated by IRE1 signaling 2, 3, 50. Therefore, it is likely that IRE1-mediated CD95 increased expression could contribute to tumour progression in cancer cells which display primary or acquired resistance to CD95L-induced cell death. Hence, to promote CD95L-induced cell death over non-apoptotic cellular outcomes, it will also be required to define tumour contexts in which additional cell death checkpoints (e.g. mediated by IAPs) should be alleviated in concert with IRE1 targeting. Additional pathological conditions have been shown to be potentially regulated by both CD95 and IRE1. For example, IRE1 inhibition prevents fibrosis in an idiopathic pulmonary fibrosis (IPF) murine model ${ }^{51,52}$. In this context, loss of CD95 promotes persistent fibrosis ${ }^{53}$, thus pointing towards this non-cancer model to also evaluate the IRE1/CD95 relationship.

Our data indicate that CD95-dependent cell death signals are oppositely regulated by RIDD and XBP1s. Such a phenomenon could therefore represent a mechanism to fine-tune life and death decisions. In addition, such dual mechanism might not be a rare occurrence. Indeed, ongoing work from the laboratory suggests that more than $10 \%$ of XBP1s target genes may also be potential RIDD substrates ${ }^{6}$. Whilst in the context of CD95 signalling this dual regulation may be an additional way to ensure timely cell death induction, it will be interesting to explore the functional consequences of modulating the other yet-to-be-validated dual targets of IRE1 signalling.

\section{Material and methods}

Cell lines, cell culture and reagents- U87, RADH85 and RADH87 (WT, DN, EV, IRE1WT or IRE1Q780*) and MDA-MB-231 WT or CD95 KO1 and 2 (previously named CD95 KO 5 and KO 9 respectively) were generated in our laboratory as described previously ${ }^{11,30-32,54}$. All cell lines were cultured in Dulbecco's modified Eagle's medium (DMEM) supplemented with $10 \%$ decomplemented FBS and $2 \mathrm{mM} \mathrm{L-glutamine} \mathrm{at}$ $37^{\circ} \mathrm{C}$ in a $5 \% \mathrm{CO}_{2}$ incubator. Modified RADH85 and 87 were cultured with $0.8 \mu \mathrm{g} / \mathrm{mL}$ or $1 \mu \mathrm{g} / \mathrm{mL}$ puromycin, respectively. All cells were regularly tested for mycoplasma absence. CD95L was produced and quantified in-house as described previously ${ }^{55}$. Thapsigargin was from Sigma-Aldrich (SML1845), Tunicamycin was from SigmaAldrich (T7765), Brefeldin A was from Selleckchem (S7046), MKC-8866 was from Selleckchem (S8875), Birinapant was from Selleckchem (S7015), MG-132 was from Selleckchem (S2619), Actinomycin D was from Sigma-Aldrich (A9415).

$R T-q P C R$ - Total RNA was extracted from cells using Trizol (Thermo-Fisher Scientific, Thermo-Fisher Scientific, 15596026) according to the manufacturer's instructions. cDNA was synthesized from the total RNA using the Maxima Reverse Transcriptase enzyme, random hexamer primers, dNTP mix and the Ribolock RNase inhibitor (Thermo-Fisher Scientific). PCR was performed on the template cDNA using Phusion High-Fidelity DNA Polymerase and dNTP mix (Thermo-Fisher Scientific). Quantitative PCR was alternatively performed for the cDNA using the SYBR® Premix Ex Taq ${ }^{\text {TM }}$ (Tli RNase H Plus) (TAKARA-Clontech) using a QuantStudio5 system (Applied Biosystems). The primer sequences used for these experiments are shown in Table 1. 
Table I

\begin{tabular}{|c|c|c|}
\hline $\begin{array}{l}\text { qPCR primers } \\
\text { (Homo } \\
\text { sapiens) }\end{array}$ & Sense (Forward 5'-3') & Anti-sense (Reverse 5'-3') \\
\hline Fas/CD95 & $\begin{array}{l}\text { 5'- } \\
\text { AATCCTGAAACAGTGGCAATA } \\
\text { AA-3' }\end{array}$ & $\begin{array}{l}5^{\prime}- \\
\text { TTTCGAACAAAGCCTTTAAC } \\
\text { TTG-3' }\end{array}$ \\
\hline GAPDH & $\begin{array}{l}5^{\prime}- \\
\text { AAGGTGAAGGTCGGAGTCAA } \\
-3^{\prime}\end{array}$ & $\begin{array}{l}5^{\prime}- \\
\text { CATGGGTGGAATCATATTGG } \\
-3^{\prime}\end{array}$ \\
\hline XBP1s & 5'-TGAGTCCGCAGCAGGTG-3' & $\begin{array}{l}\text { 5'- } \\
\text { GCTGGCAGGCTCTGGGGAA } \\
\text { G-3' }\end{array}$ \\
\hline $\begin{array}{l}\text { Fas } \\
\text { (CAACAA ; } \\
\text { first site) }\end{array}$ & $\begin{array}{l}\text { 5'-CGG AGT TGG GGA AGC } \\
\text { TCT TT-3' }\end{array}$ & $\begin{array}{l}\text { 5'-TGA TGT CAG TCA CTT } \\
\text { GGG CA-3' }\end{array}$ \\
\hline $\begin{array}{l}\text { Fas } \\
\text { (CTGCAT ; } \\
\text { first site) }\end{array}$ & $\begin{array}{l}\text { 5'-GGA CCC TCC TAC CTC } \\
\text { TGG TT-3' }\end{array}$ & $\begin{array}{l}\text { 5'-GAG GAC AGG GCT TAT } \\
\text { GGC AG-3' }\end{array}$ \\
\hline $\begin{array}{l}\text { Fas } \\
\text { (CAACAA; } \\
\text { second site) } \\
\end{array}$ & $\begin{array}{l}\text { 5'-TGT CCA AGA CAC AGC } \\
\text { AGA ACA-3' }\end{array}$ & $\begin{array}{l}\text { 5'-CCA AGC AGT ATT TAC } \\
\text { AGC CAG C-3' }\end{array}$ \\
\hline $\begin{array}{l}\text { Fas } \\
\text { (CTGCAT ; } \\
\text { second site) } \\
\end{array}$ & $\begin{array}{l}\text { 5'-AGA CCT CTT CCA AAC } \\
\text { AGC ACA-3' }\end{array}$ & $\begin{array}{l}\text { 5'-AAA GAA AGG TGG CCC } \\
\text { ACT GG-3' }\end{array}$ \\
\hline $\begin{array}{l}\text { Fas } \\
\text { (CTGCAG) }\end{array}$ & $\begin{array}{l}\text { 5'-CAC CCA AAG GTC ACC } \\
\text { CAT GA-3' }\end{array}$ & $\begin{array}{l}\text { 5'-CCC TGT GGG CAT GCC } \\
\text { AAT AA-3' }\end{array}$ \\
\hline
\end{tabular}

In vitro RNA cleavage assay-

The predicted CD95 mRNA structure was obtained using the RNAfold Web server ${ }^{56}$. Different concentrations of recombinant IRE1(Sino Biological) were incubated with 2 $\mu \mathrm{g}$ of RNA extracts from U87 cell line, 2mM DTT (Merck, D3801), 20 mM ATP (Merck, A2383) in $20 \mathrm{mM}$ Tris, $500 \mathrm{mM} \mathrm{NaCl}, 10 \%$ gly, $\mathrm{pH} 7.4$ for 1 hour at $37^{\circ} \mathrm{C}$. After the incubation, the resulting samples were used to perform qRT-PCR as described above.

Cell and tissue lysis and western blot- For cell lines: cells seeded in 6 well plates were treated as indicated in the figure legends and lysed in 200-250 $\mu \mathrm{L}$ ice-cold RIPA buffer (Tris- $\mathrm{HCl} 50 \mathrm{mM}$, ph7.4, NaCl $150 \mathrm{mM}$, EDTA 2 mM, Sodium Deoxycholate 0.5\%, Triton 1\%; SDS $0.1 \%$ ) per well, including proteases (Protease inhibitor cocktail, Sigma-Aldrich, P8340) and phosphatase inhibitors (Phosphatase inhibitor cocktail 2, Sigma-Aldrich, P5726). After 10 minutes incubation on ice, lysates were briefly sonicated and cleared by centrifugation $\left(15500 \mathrm{~g}, 30 \mathrm{~min}, 4^{\circ} \mathrm{C}\right)$. Lysates were collected and protein quantified using Pierce ${ }^{\mathrm{TM}} \mathrm{BCA}$ protein assay (Thermo scientific). Laemmli sample buffer (BIO-RAD, 1610747) with 2-mercaptoethanol (BIO-RAD, 1610710) was added to the samples which were heated $\left(95^{\circ} \mathrm{C}, 5 \mathrm{~min}\right)$. For liver tissue: tissue pieces (300-450 mg) were flash-frozen using liquid nitrogen upon collection. Tissues were homogenized with a Precellys Lysing kit (Bertin Ref. P000911) according to the manufacturer's instructions. For western blot analyses of cell and tissue lysates, 10-50 $\mu \mathrm{g}$ protein was loaded on $10 \%$ acrylamide home-made or gradient (4-15\% acrylamide) 
commercial (BIO-RAD, 4561086) Tris-Glycine-SDS gels along a protein ladder (PageRuler prestained protein ladder, Thermo scientific, 26617). Proteins were then transferred onto nitrocellulose membranes using the turbo transfer system (BIO-RAD). Membranes were saturated with TBS with 0.5\% Tween-20 and 5\% Milk for 1 hour prior to overnight $4^{\circ} \mathrm{C}$ incubation with the indicated primary antibody diluted $1 / 1000^{\circ}$ in TBSTween-5\%BSA $0.025 \%$ Sodium Azide. HRP-coupled secondary antibodies, used at $1 / 5000^{\circ}$ in TBS-Tween-5\% milk, were from Southern Biotech. Signal Fire ${ }^{\mathrm{TM}} \mathrm{ECL}$ reagent (Cell signaling) or ECL Revelblot intense (OZYme, OZYB002-1000) was used and chemiluminescence signal was detected using G:Box Chemi XX6 imager from Syngene. For quantification, the Image $\mathrm{J}$ software was used ${ }^{57}$.

The following primary antibodies were used for western blot: CD95 (Cell signaling, 4233), Actin (Sigma-Aldrich, A5316), IRE1 (Cell signaling, 3294 and Santa Cruz, 390960), XBP1s (Biolegend, 647502), Caspase-8 (Adipogen, AG-20B-0057-C100, Caspase-3 (Cell signaling, 14220), Cleaved caspase-3 (Cell signaling, 9661).

Flow cytometry- To evaluate CD95 expression at the cell surface, cells (U87, RADH85 or RADH87) were seeded in 6 well plates (250000 cells/well). 24 hours later, cells were harvested and stained using Zombie-violet ${ }^{\mathrm{TM}}$ (Biolegend, 423113) diluted in PBS according to the manufacturer protocol. Then, samples were saturated with PBS containing 1\% BSA and 1\% FCS and human FcR-blocking reagent (Miltenyi Biotec, 130-059-901) for 15 minutes at $4^{\circ} \mathrm{C}$ prior to labelling using an anti-human CD95-APC antibody (clone DX2, Miltenyi Biotec, 130-117-701) or corresponding IgG1-APC isotype (clone IS5-21F5, Miltenyi Biotec, 130-113-196) for 30 minutes at $4^{\circ} \mathrm{C}$. Following two washes with the saturation buffer, cells were resuspended in PBS and analysed using a Novocyte cytometer (Acea Biosciences). CD95 cell surface expression was analysed after gating on viable singlets. Results are represented as a ratio of Median Intensity Fluorescence values for anti-CD95-stained over isotypestained samples.

Transfection- The cells indicated in the legend were seeded in 6 well plates at 250000 cells per well. For knockdown experiments, cells were transfected 24 hours after seeding using LipoRNAimax (Invitrogen, 13778075) according to the manufacturer protocol with $80 \mathrm{nM}$ siRNA (On-target plus Non-targeting or Human FAS pool, Horizon Discovery, D-001810-10-05 or L-003776-00-0005, respectively). 24 hours after transfection, cells were reseeded in 48 well plate and 6 well plate for viability and western blot analysis respectively. 24 hours after reseeding, cells were either lysed (for western blot) or treated as described for viability assay. For over-expression experiments, cells were transfected 24 hours after seeding using $2.5 \mu \mathrm{g}$ DNA per well and lipofectamine 3000 (Invitrogen, L3000008) according to the manufacturer protocol. pCMV5-Flag-XBP1s was a gift from Qingbo Xu \& Lingfang Zeng (Addgene plasmid \# $63680)^{58} .24$ hours after transfection, cells were reseeded in 48 well plate. 24 hours after reseeding, cells were treated as described for viability assay.

Viability assay- Cells seeded in 48 well plates (20000 cells per well) were treated as indicated in the figure legends. $500 \mu \mathrm{g} / \mathrm{mL}$ MTT (Invitrogen, M6494) was added to the medium for the last two hours of treatment. Medium was then removed and the formazan crystals were solubilized using DMSO. Reading of the absorbance was performed at $560 \mathrm{~nm}$ using a Tecan Infinite F200 Pro reader. Results are expressed as $\%$ of viability, with $100 \%$ being untreated cells for each cell line or experimental condition. 
In vivo experiments- All in vivo experiments described in this study have been approved by the Comité d'éthique de l'Université de Rennes 1 and the French ministry of education, research and innovation under the licence APAFIS \#322412021061714545440. These experiments were performed at the ARCHE-BIOSIT UMS 34380 (Rennes). 8-weeks old C57BL/6rJ male mice were obtained from Janvier. Mice were fasted on day 1 at $12 \mathrm{pm}$ for both experiments. Volumes injected intraperitoneally did not exceed $250 \mu \mathrm{L}$ for both experiments. For experiment 1 , intraperitoneal injections of MKC-8866 (or equivalent volume of DMSO) diluted in PBS to $10 \mathrm{mg} / \mathrm{mL}$ were performed at $6 \mathrm{pm}$ on day 1 and 8 am on day 2 and mice were culled at $12 \mathrm{pm}$ on day 2. For IHC analyses, a liver piece (around $200 \mathrm{mg}$ ) was fixed in paraformaldehyde $4 \%$ solution. The rest of the liver was rapidly cut in small pieces prior to flash-freezing using liquid nitrogen for western blot analysis. For experiment 2 , intra-peritoneal injections of MKC-8866 (or equivalent volume of DMSO) diluted in PBS to $10 \mathrm{mg} / \mathrm{mL}$ were performed at $12 \mathrm{pm}$ on day $1,6 \mathrm{pm}$ on day 1 and 8 am on day 2 . At $12 \mathrm{pm}$ on day 2 , an intra-peritoneal injection of $0,15 \mathrm{mg} / \mathrm{kg}$ of anti-CD95 antibody Jo2 (BD Biosciences, 554254) or corresponding isotype control (BD Biosciences, 553961) was performed. Mice were culled 6 hours later and liver samples collected as described for experiment 1.

Statistical analyses- All statistical analyses were performed using Graphpad PRISM (v9) and are described in the figure legends.

\section{RNAseq and TCGA analyses}

For each sample, the XBP1s and RIDD activity scores were calculated using the 38 genes from the IRE1 signature ${ }^{11}$. The expression values of the 38 transcripts were extracted from the normalized count matrix of the 67 TNBC transcriptomes from a local cohort (GSE182021) and the 45 TCGA-GBM transcriptomes. Samples were then classified as high or low for XBP1s and RIDD activities according to the median. Then CD95 mRNA expression z-score was calculated for each sample and plotted according to the XBP1s and RIDD activities in both TNBC (TN) and GBM (GB) datasets.

\section{Immunohistochemistry (IHC) and quantification}

For IHC, samples were fixed in PFA 4\% for at least 24 hours, embedded in paraffin at least 12 hours and sliced $(4 \mu \mathrm{m})$ using a Leica microtome on Superfrost Plus slides (VWR, 631-0108) prior to drying at $60^{\circ} \mathrm{C}$ for 1 hour. The immunochemistry experiments were performed using the Discovery XT machine (Roche) and the Chromo-Map DAB kit (Roche). The following primary antibodies: cleaved caspase-3, Cell Signalling, 9661, diluted 1/300; CD95, R\&D systems, AF435, diluted 1/50 in antibody diluent (NB23-00171-1, NeoBiotech) were incubated for 1 hour at $37^{\circ} \mathrm{C}$. To perform the analysis, glass slides were digitized with the scanner Nanozoomer 2.0-RS Hamamatsu. The quantifications of CD95 expression and cleaved caspase-3 staining were carried out using the NIS-Elements software (Nikon) and averaged from 5 fields covering $4 \mathrm{~mm}^{2}$ in total per liver.

\section{Acknowledgements}

We thank all the members of the U1242 for fruitful scientific discussions. We thank Drs Michel Samson and Jacques Le Seyec for their advice on the in vivo liver damage model. We thank the BIOSIT H2P2 platform for immunohistochemistry, in particular Gevorg Ghukasyan, and the BIOSIT Animal facility ARCHE (https://biosit.univrennes1.fr/). This work was funded by grants from Fondation ARC (PDF20171206671) 
and Fondation de France to EL, INCa PLBIO 2018, 2019 grants to EC, PJA20181207700 (Fondation ARC) grant to EL and MLG, Associations la Vannetaise et la Josselinaise des femmes funding to MLG and Centre Eugène Marquis (EL, EC, MLG, TA and SM).

\section{Author contributions}

Conceptualisation: EC, EL; Formal analysis: AP, DPR, EL, MLG; Funding acquisition: EC, EL, MLG; Investigation: AP, DPR, EL, RP, MLG, SM, TA; Methodology: RP; Project administration: EL; Supervision: EC, EL; Validation: DPR, EL; Visualisation: AP, DPR, EL, MLG; Writing-original draft: EL; Writing-review and editing: AP, TA, DPR, $E C, E L, M L G, S M$.

\section{Conflict of interest}

EC is a founder of Cell Stress Discoveries Ltd and Thabor Therapeutics. The authors do not declare any conflict of interest.

\section{References}

1. Von Karstedt, S., Montinaro, A. \& Walczak, H. Exploring the TRAILs less travelled: TRAIL in cancer biology and therapy. Nature Reviews Cancer (2017).

2. Rossin, A., Miloro, G. \& Hueber, A.O. TRAIL and FasL Functions in Cancer and Autoimmune Diseases: Towards an Increasing Complexity. Cancers 11 (2019).

3. Risso, V., Lafont, E. , Le Gallo,M. Therapeutic approaches targeting CD95L/CD95 signaling in cancer and autoimmune diseases. Cell Death Disease In press (2022).

4. Almanza, A. et al. Endoplasmic reticulum stress signalling - from basic mechanisms to clinical applications. Febs j 286, 241-278 (2019).

5. McGrath, E.P., Centonze, F.G., Chevet, E., Avril, T. \& Lafont, E. Death sentence: The tale of a fallen endoplasmic reticulum. Biochimica Et Biophysica Acta Bba - Mol Cell Res, 119001 (2021).

6. Obacz, J. et al. Novel IRE1-dependent proinflammatory signaling controls tumor infiltration by myeloid cells. 533018 (2020).

7. Doultsinos, D. et al. Control of glioblastoma differentiated-to-stem cell reprogramming by IREla/XBPIs signaling. Biorxiv, 594630 (2019).

8. Drachsler, M. et al. CD95 maintains stem cell-like and non-classical EMT programs in primary human glioblastoma cells. Cell Death Dis 7, e2209 (2016).

9. Kleber, S. et al. Yes and PI3K bind CD95 to signal invasion of glioblastoma. Cancer Cell 13, 235-248 (2008).

10. Le Reste, P.J. et al. Local intracerebral Inhibition of IRE1 by MKC8866 sensitizes glioblastoma to irradiation/chemotherapy in vivo. 841296 (2020).

11. Lhomond, S. et al. Dual IRE1 RNase functions dictate glioblastoma development. EMBO Mol Med 10 (2018).

12. Chen, X. et al. XBP1 promotes triple-negative breast cancer by controlling the HIF1alpha pathway. Nature 508, 103-107 (2014).

13. Fritsche, $\mathrm{H}$. et al. TRAIL-R2 promotes skeletal metastasis in a breast cancer xenograft mouse model. Oncotarget 6, 9502-9516 (2015).

14. Logue, S.E. et al. Inhibition of IRE1 RNase activity modulates the tumor cell secretome and enhances response to chemotherapy. Nat Commun 9, 3267 (2018).

15. Harnoss, J.M. et al. IRE1a Disruption in Triple-Negative Breast Cancer Cooperates with Antiangiogenic Therapy by Reversing ER Stress Adaptation 
and Remodeling the Tumor Microenvironment. Cancer Res 80, 2368-2379 (2020).

16. Le Thomas, A. et al. Noncanonical mRNA decay by the endoplasmic-reticulum stress sensor IRE1a promotes cancer-cell survival. 2021.2003.2016.435520 (2021).

17. Maurel, M., Chevet, E., Tavernier, J. \& Gerlo, S. Getting RIDD of RNA: IRE1 in cell fate regulation. Trends Biochem Sci 39, 245-254 (2014).

18. Lafont, E. Stress management : Death Receptor Signalling and its Cross-talks with the Unfolded Protein Response in Cancer. Cancers 12, 1113 (2020).

19. Stöhr, D., Jeltsch, A. \& Rehm, M. TRAIL receptor signaling: From the basics of canonical signal transduction toward its entanglement with ER stress and the unfolded protein response. International review of cell and molecular biology 351, 57-99 (2020).

20. Li, T. et al. DDIT3 and KAT2A Proteins Regulate TNFRSF10A and TNFRSF10B Expression in Endoplasmic Reticulum Stress-mediated Apoptosis in Human Lung Cancer Cells. J Biol Chem 290, 11108-11118 (2015).

21. Lam, M., Lawrence, D.A., Ashkenazi, A. \& Walter, P. Confirming a critical role for death receptor 5 and caspase- 8 in apoptosis induction by endoplasmic reticulum stress. Cell Death Differ 25, 1530-1531 (2018).

22. Lu, M. et al. Opposing unfolded-protein-response signals converge on death receptor 5 to control apoptosis. Science 345, 98-101 (2014).

23. He, Q. et al. Endoplasmic reticulum calcium pool depletion-induced apoptosis is coupled with activation of the death receptor 5 pathway. Oncogene 21, 26232633 (2002).

24. Yamaguchi, H. \& Wang, H.G. CHOP is involved in endoplasmic reticulum stress-induced apoptosis by enhancing DR5 expression in human carcinoma cells. J Biol Chem 279, 45495-45502 (2004).

25. Cazanave, S.C. et al. Death receptor 5 signaling promotes hepatocyte lipoapoptosis. J Biol Chem 286, 39336-39348 (2011).

26. Jiang, C.C. et al. Tunicamycin sensitizes human melanoma cells to tumor necrosis factor-related apoptosis-inducing ligand-induced apoptosis by upregulation of TRAIL-R2 via the unfolded protein response. Cancer Res 67, 5880-5888 (2007).

27. Iurlaro, R. et al. Glucose Deprivation Induces ATF4-Mediated Apoptosis through TRAIL Death Receptors. Mol Cell Biol 37 (2017).

28. Iurlaro, R. \& Munoz-Pinedo, C. Cell death induced by endoplasmic reticulum stress. Febs $j$ 283, 2640-2652 (2016).

29. Lam, M., Marsters, S.A., Ashkenazi, A. \& Walter, P. Misfolded proteins bind and activate death receptor 5 to induce apoptosis during unresolved endoplasmic reticulum stress. Elife 9 (2020).

30. Pluquet, $\mathrm{O}$. et al. Posttranscriptional Regulation of PER1 Underlies the Oncogenic Function of IREa. Cancer research 73, 4732-4743 (2013).

31. Nguyên, D.T. et al. Nck-dependent Activation of Extracellular Signal-regulated Kinase-1 and Regulation of Cell Survival during Endoplasmic Reticulum Stress. Molecular biology of the cell 15, 4248-4260 (2004).

32. Avril, T. et al. Human glioblastoma stem-like cells are more sensitive to allogeneic NK and T cell-mediated killing compared with serum-cultured glioblastoma cells. Brain pathology (Zurich, Switzerland) 22, 159-174 (2012).

33. Volkmann, K. et al. Potent and selective inhibitors of the inositol-requiring enzyme 1 endoribonuclease. J Biol Chem 286, 12743-12755 (2011). 
34. Moore, K. \& Hollien, J. Ire1-mediated decay in mammalian cells relies on mRNA sequence, structure, and translational status. Molecular biology of the cell 26, 2873-2884 (2015).

35. Oikawa, D., Tokuda, M., Hosoda, A. \& Iwawaki, T. Identification of a consensus element recognized and cleaved by IRE1 alpha. Nucleic Acids Res 38, 62656273 (2010).

36. Yoshida, H., Matsui, T., Yamamoto, A., Okada, T. \& Mori, K. XBP1 mRNA is induced by ATF6 and spliced by IRE1 in response to ER stress to produce a highly active transcription factor. Cell 107, 881-891 (2001).

37. Voutetakis, K.D., D.; Vlachavas, E-I., Leonidas, DD.; Chevet, E.; Chatzioannou, A. RNA sequence motif and structure in IRE1-mediated cleavage. (In preparation).

38. Geserick, P. et al. Cellular IAPs inhibit a cryptic CD95-induced cell death by limiting RIP1 kinase recruitment. J Cell Biol 187, 1037-1054 (2009).

39. Jost, P.J. et al. XIAP discriminates between type I and type II FAS-induced apoptosis. Nature 460, 1035-1039 (2009).

40. Condon, S.M. et al. Birinapant, a smac-mimetic with improved tolerability for the treatment of solid tumors and hematological malignancies. J Med Chem 57, 3666-3677 (2014).

41. Benetatos, C.A. et al. Birinapant (TL32711), a bivalent SMAC mimetic, targets TRAF2-associated clAPs, abrogates TNF-induced NF-kB activation, and is active in patient-derived xenograft models. Molecular cancer therapeutics 13, 867-879 (2014).

42. Filliol, A. et al. RIPK1 protects hepatocytes from death in Fas-induced hepatitis. Sci Rep 7, 9205 (2017).

43. Stöhr, D. et al. Stress-induced TRAILR2 expression overcomes TRAIL resistance in cancer cell spheroids. Cell Death Differ 27, 3037-3052 (2020).

44. Sullivan, G.P. et al. TRAIL Receptors Serve as Stress-Associated Molecular Patterns to Promote ER-Stress-Induced Inflammation. Dev Cell 52, 714730.e715 (2020).

45. Hellwig, C.T. et al. Proteasome inhibition triggers the formation of TRAIL receptor 2 platforms for caspase-8 activation that accumulate in the cytosol. Cell Death Differ 29, 147-155 (2022).

46. Timmins, J.M. et al. Calcium/calmodulin-dependent protein kinase II links ER stress with Fas and mitochondrial apoptosis pathways. J Clin Invest 119, 29252941 (2009).

47. Miani, M., Colli, M.L., Ladrière, L., Cnop, M. \& Eizirik, D.L. Mild endoplasmic reticulum stress augments the proinflammatory effect of IL-1 $\beta$ in pancreatic rat $\beta$-cells via the IRE1 1 /XBP1s pathway. Endocrinology 153, 3017-3028 (2012).

48. Singh, N. et al. Impaired Death Receptor Signaling in Leukemia Causes Antigen-Independent Resistance by Inducing CAR T-cell Dysfunction. Cancer Discov 10, 552-567 (2020).

49. Upadhyay, R. et al. A Critical Role for Fas-Mediated Off-Target Tumor Killing in T-cell Immunotherapy. Cancer Discov 11, 599-613 (2021).

50. Annibaldi, A. \& Walczak, H. Death Receptors and Their Ligands in Inflammatory Disease and Cancer. Cold Spring Harb Perspect Biol 12, a036384 (2020).

51. Thamsen, M. et al. Small molecule inhibition of IRE1 a kinase/RNase has antifibrotic effects in the lung. PLoS One 14, e0209824 (2019). 
52. Auyeung, V.C. et al. IRE1a drives lung epithelial progenitor dysfunction to establish a niche for pulmonary fibrosis. American journal of physiology. Lung cellular and molecular physiology (2022).

53. Redente, E.F. et al. Loss of Fas signaling in fibroblasts impairs homeostatic fibrosis resolution and promotes persistent pulmonary fibrosis. $\mathrm{JCl}$ insight 6 (2020).

54. Guégan, J.P. et al. CD95/Fas suppresses NF-kB activation through recruitment of KPC2 in a CD95L/FasL-independent mechanism. iScience 24, 103538 (2021).

55. Risso, V., Thomas, M., Guevel, B., Lavigne. R, Com, E., Martin, S., Pineau, C., Negroni, L. , Lafont, E., Chevet, E., Eriksson L.A., Le Gallo,M. Metalloproteasemediated cleavage of CD95 Ligand. Submitted.

56. Lorenz, R. et al. ViennaRNA Package 2.0. Algorithms for molecular biology: AMB 6, 26 (2011).

57. Schneider, C.A., Rasband, W.S. \& Eliceiri, K.W. NIH Image to ImageJ: 25 years of image analysis. Nat Methods 9, 671-675 (2012).

58. Zeng, L. et al. Sustained activation of XBP1 splicing leads to endothelial apoptosis and atherosclerosis development in response to disturbed flow. Proc Natl Acad Sci U S A 106, 8326-8331 (2009). 
$\begin{array}{llllllll}\text { A } & \text { U87 } & \text { RADH } 85 & \text { RADH } 87 & \text { B } & \text { U87 } & \text { RADH } 85 & \text { RADH } 87\end{array}$
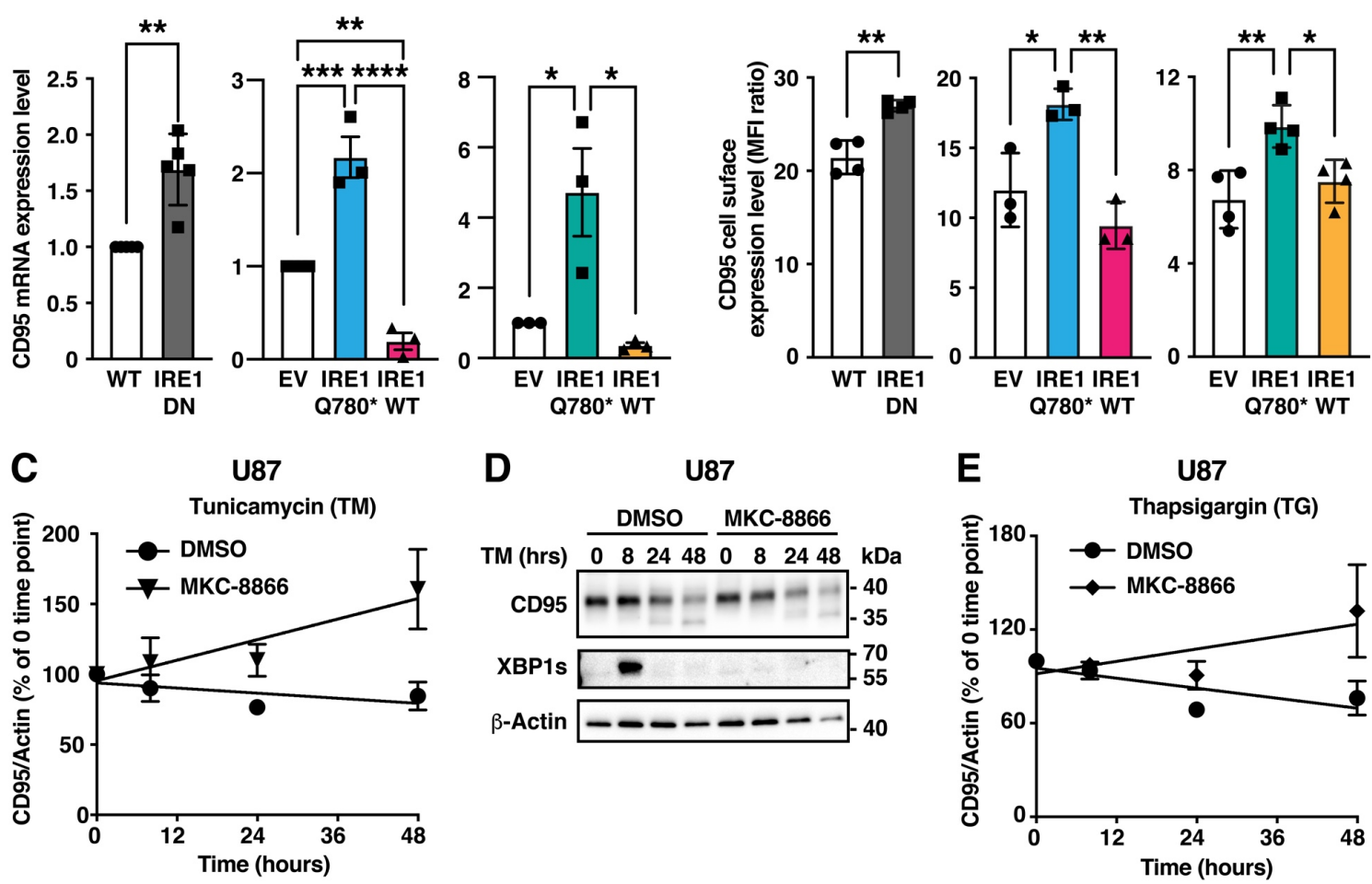

D U87
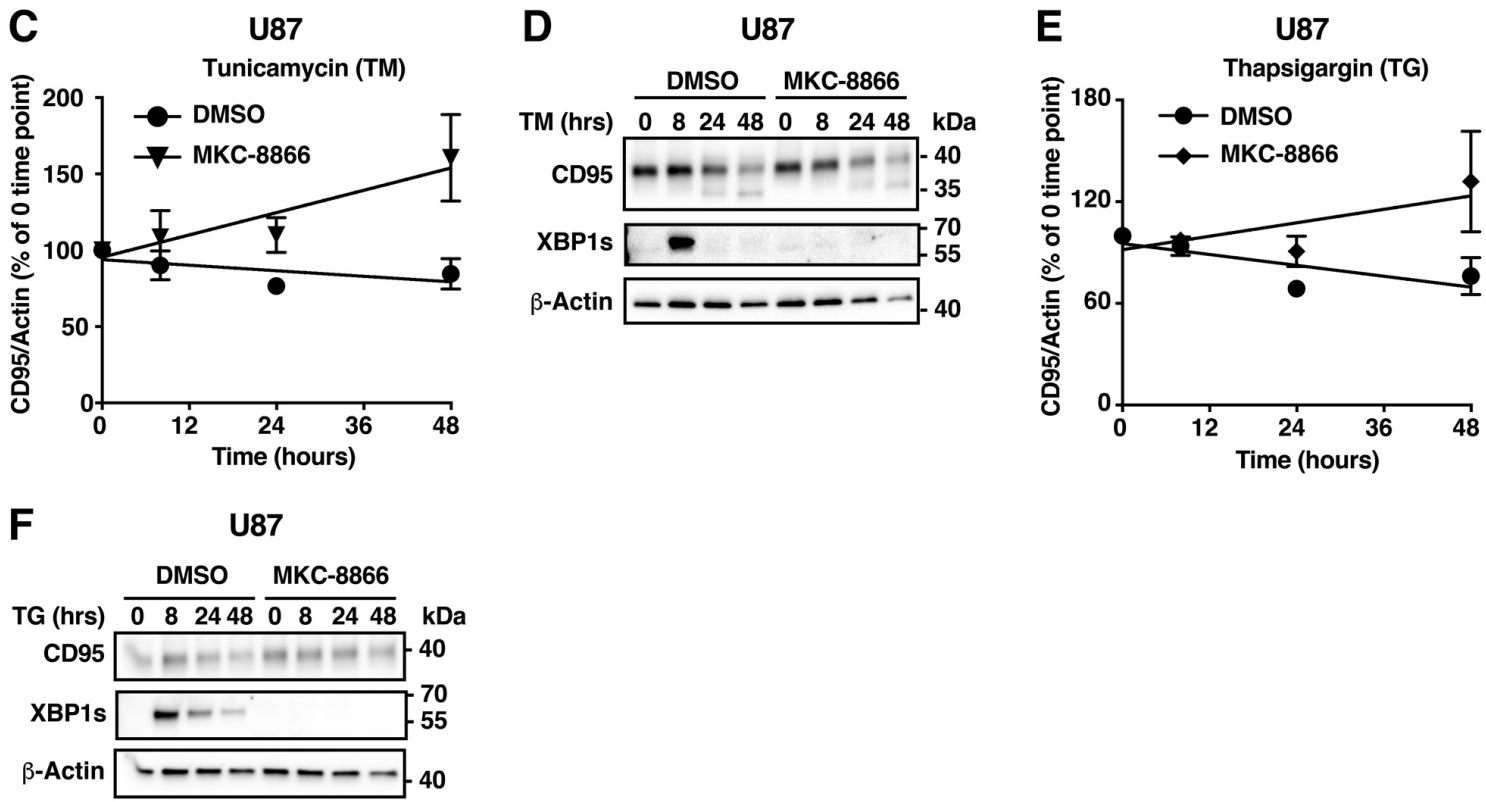

Figure 1. IRE1 regulates CD95 expression in TNBC and GB cells

A. mRNA was extracted from WT or IRE1 DN U87 cells and from empty vector (EV), IRE1WT- or IRE1Q780*-expressing RADH85 and RADH87 cells. CD95 mRNA was quantified by RT-qPCR and normalized to GAPDH. Mean \pm SEM, $n=3-5 .{ }^{* *} p<0.01$, ${ }^{* * *} p<0.001,{ }^{* * *} p<0.0001$ unpaired t-test with Welch's correction (U87) or one-way ANOVA with Tukey multiple comparison correction for RADH85 and RADH87. B. CD95 cell surface level expression was evaluated by flow cytometry. Mean of MFI ratio $\pm \mathrm{SD}, \mathrm{n}=3 .{ }^{*} \mathrm{p}<0.05,{ }^{* *} \mathrm{p}<0.01$, unpaired t-test for U87; one-way ANOVA with Tukey multiple comparison correction for RADH85 and RADH87. C, D U87 cells pre-treated for 2 hours with MKC-8866 $(30 \mu \mathrm{M})$ as indicated were further treated with $500 \mathrm{ng} / \mathrm{mL}$ tunicamycin for the indicated times. Lysates were analysed by western blot. $\mathbf{C}$. Quantification for three independent experiments is depicted. Mean \pm SEM. D. One representative experiment out of three is shown. E, F. U87 cells pre-treated for 2 hours with $\mathrm{MKC}-8866(30 \mu \mathrm{M})$ as indicated were further treated with $50 \mathrm{nM}$ thapsigargin for the indicated times. Lysates were analysed by western blot. E. Quantification for three independent experiments is depicted. Mean \pm SEM. F. One representative experiment out of three is shown. 

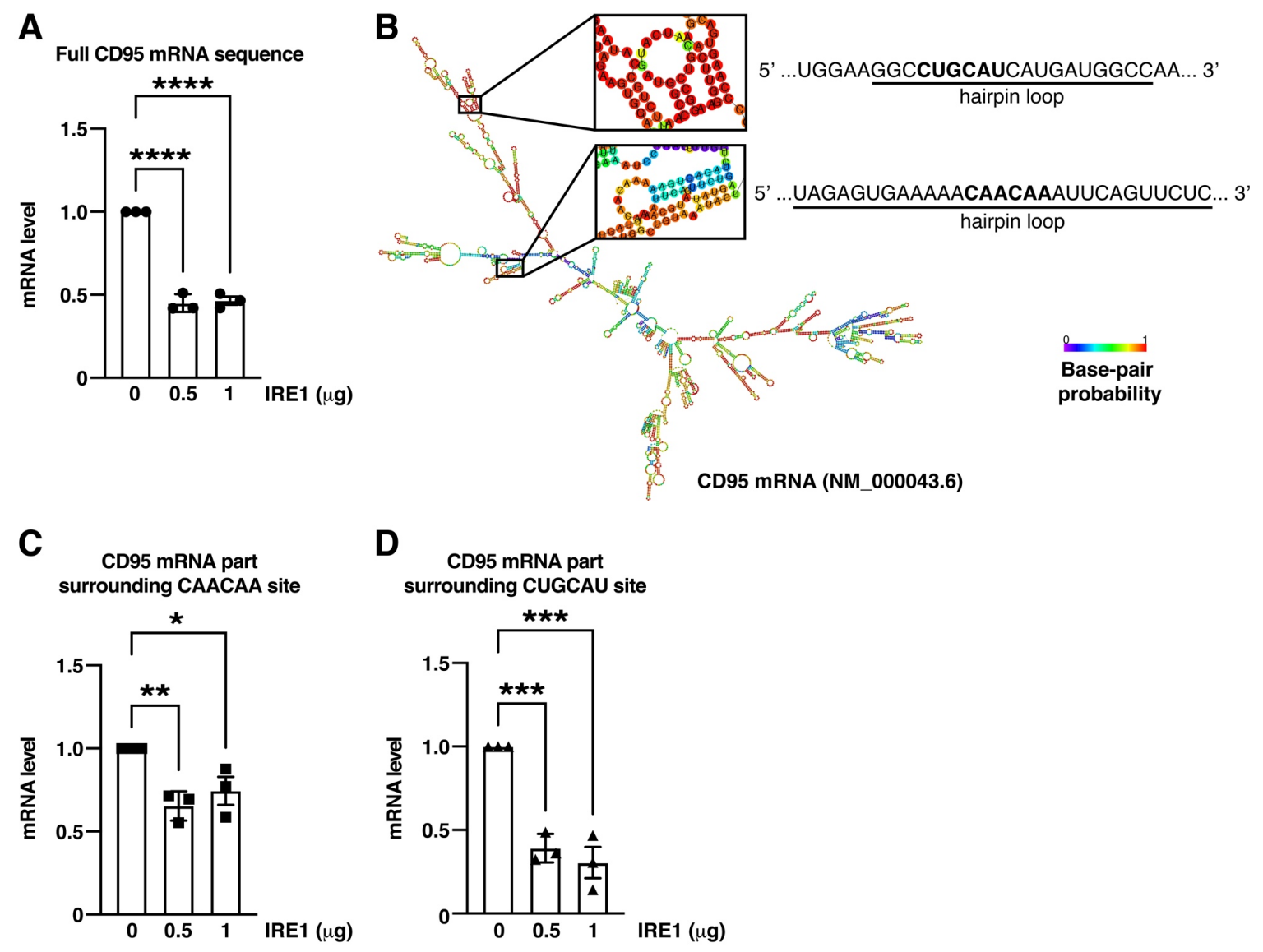

Figure 2. IRE1 cleaves CD95 mRNA in vitro

A. RNA $(2 \mu \mathrm{g})$ extracted from U87 cells was incubated with the indicated amounts of recombinant IRE1 for 1 hour. CD95 mRNA was then quantified by RT-qPCR and normalized to GAPDH. Mean \pm SEM, $n=3$. One-way ANOVA with Dunnett multiple comparison correction, ${ }^{* * * *} p<0.0001$. B. Predicted folded structure of CD95 mRNA. The two predicted cleavage sites within hairpin loops are highlighted. C, D. RNA $(2 \mu \mathrm{g})$ extracted from U87 cells was incubated with the indicated amounts of recombinant IRE1 for 1 hour. 136-bp (C) and 121-bp (D) parts of CD95 mRNA surrounding the indicated potential cleavage sites were then quantified by RT-qPCR and normalized to GAPDH. Mean \pm SEM, $n=3$. One-way ANOVA with Dunnett multiple comparison correction, ${ }^{*} p<0.05,{ }^{* *} p<0.01,{ }^{* * *} p<0.001$. 
$A^{-}$
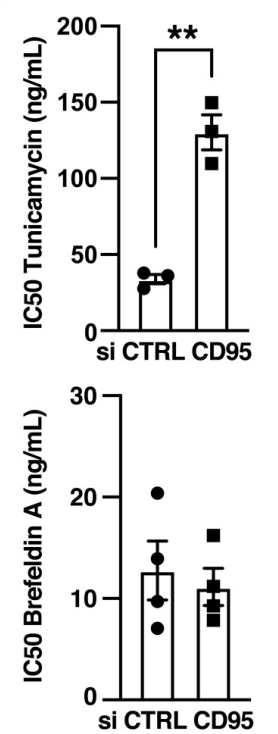

C

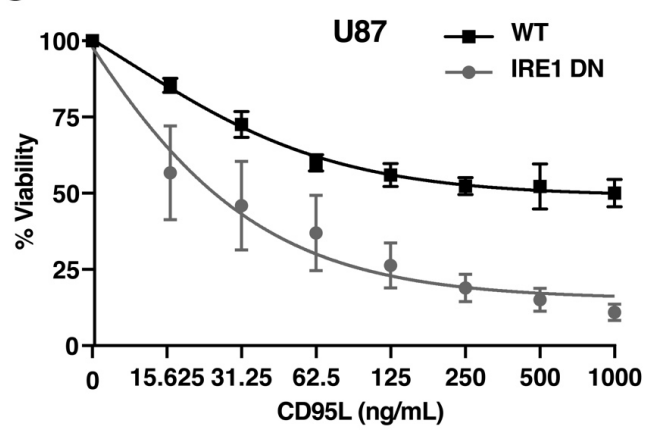

$\mathbf{E}$
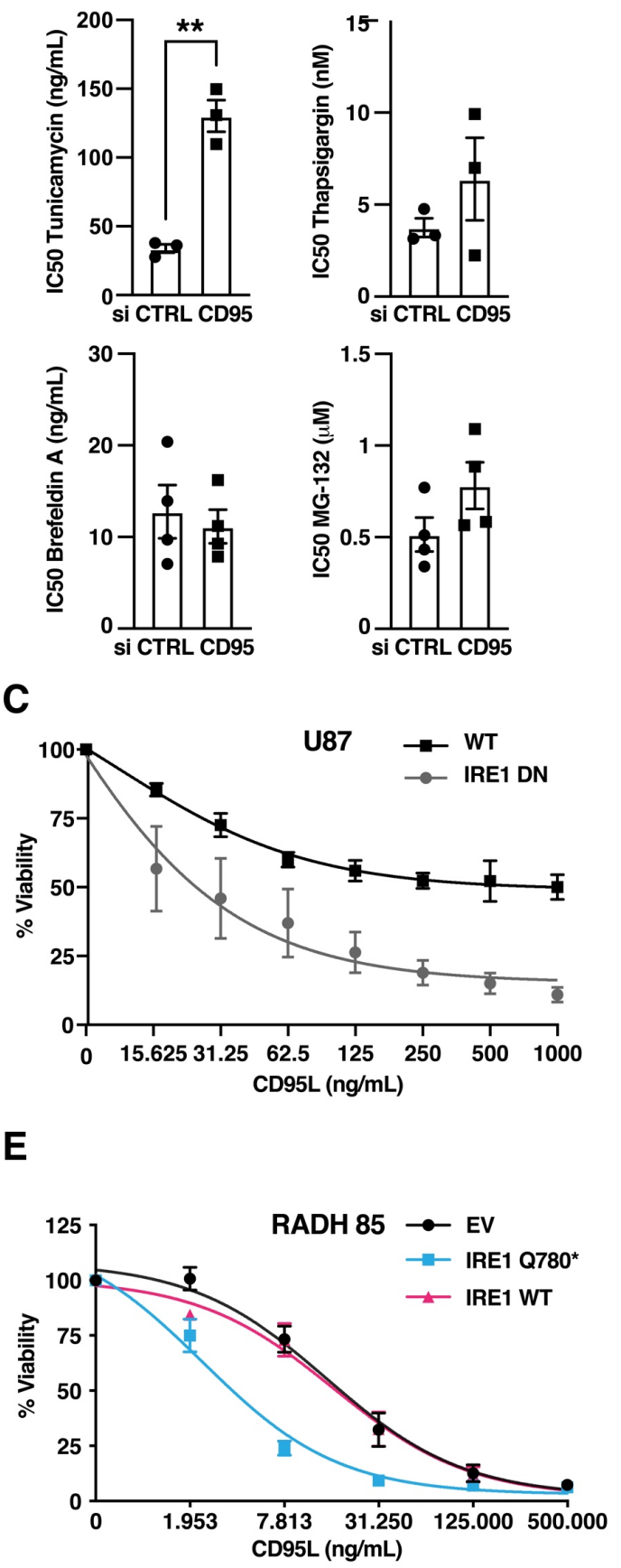

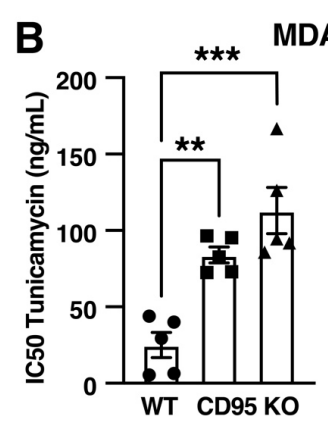

MDA-MB-231

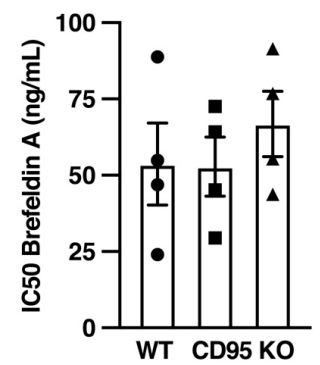

D

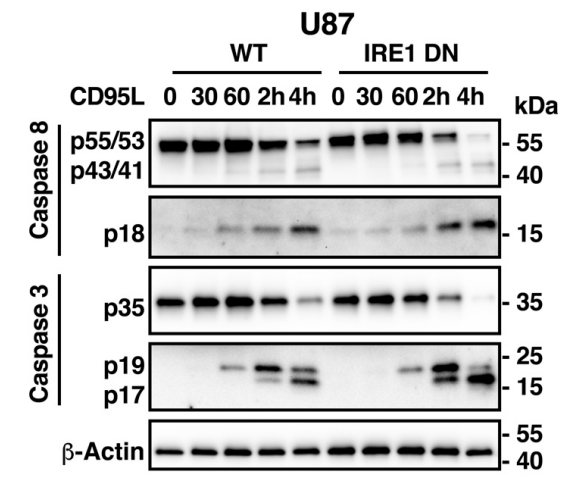

$\mathbf{F}$

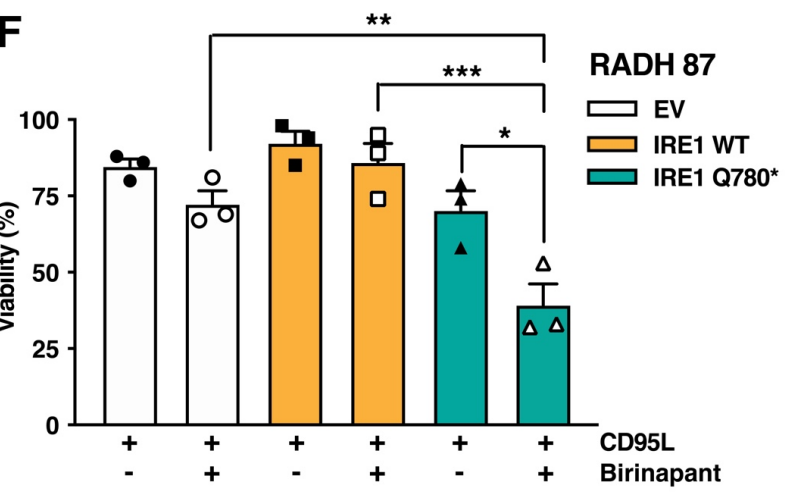

Figure 3 CD95 is not a universal determinant of ER-stress induced cell death whilst IRE1 RNase activity limits CD95L-induced cell death

A. U87 were transfected with siRNA control or targeting CD95. 48 hours later, cells were treated for 48 hours with the indicated ER stress inducers. Viability was determined using an MTT assay. The relative IC50 calculated for each independent experiment is represented (see also Fig S2A). ${ }^{* *} p<0.01$, unpaired t-test B. MDA-MB231 WT or CD95 KO clones were treated for 48 hours with the indicated ER stress inducers. Viability was determined using an MTT assay and relative IC50 calculated for each independent experiment (see also Fig S2B). ${ }^{* *} p<0.01$, ${ }^{* * *} p<0.001$, one-way ANOVA with Tukey multiple comparison correction C. U87 WT or expressing IRE1DN were treated with the indicated concentrations of CD95L for 24 hours. Viability was determined using MTT assay and normalized to untreated cell values. Mean \pm SEM of 3 independent experiments. D. U87 WT or expressing IRE1DN were treated with 250 
$\mathrm{ng} / \mathrm{mL}$ CD95L for the indicated times. Lysates were analysed by western blot. One experiment representative of three independent ones is shown. E. RADH85 control (EV), stably expressing IRE1Q780* or IRE1WT were treated with the indicated concentrations of CD95L for 24 hours. Viability was determined using MTT assay and normalised to untreated cells values. Mean \pm SEM of 3 independent experiments. $F$. RADH87 control (EV), stably expressing IRE1Q780* or IRE1WT were pre-treated with $50 \mathrm{nM}$ Birinapant and further treated with $1 \mu \mathrm{g} / \mathrm{mL}$ CD95L for 24 hours. Viability was determined using MTT assay and normalised to untreated cells values. Mean \pm SEM of 3 independent experiments. ${ }^{* *} p<0.05,{ }^{* *} p<0.01,{ }^{* * *} p<0.001$, one-way ANOVA with Tukey multiple comparison correction. 


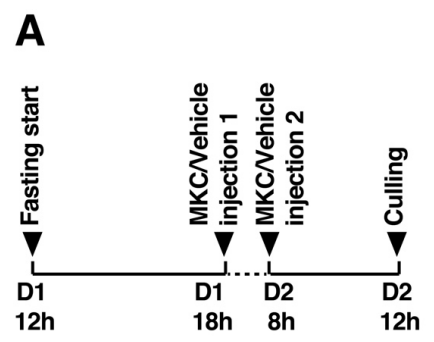

B
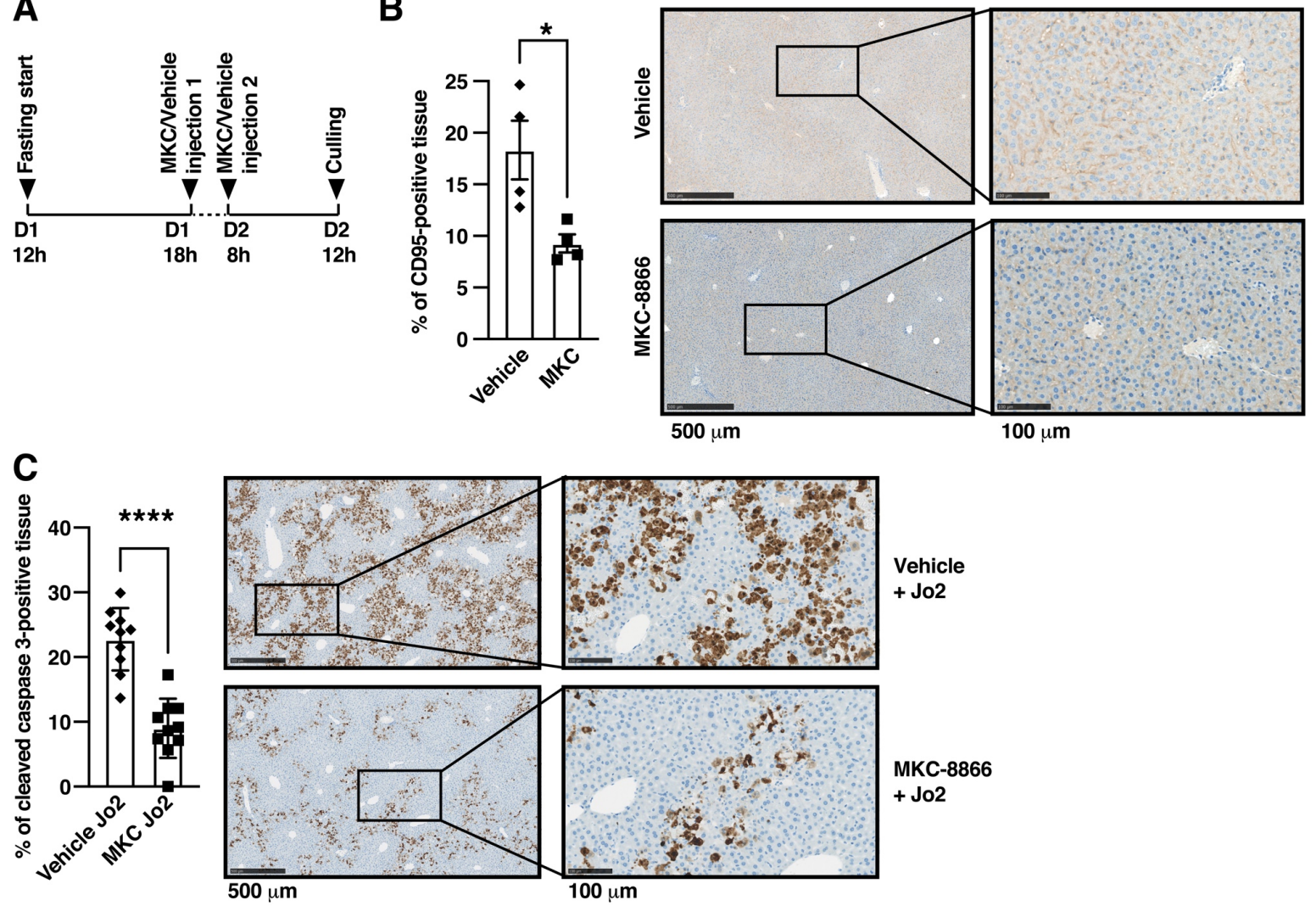

Figure 4 IRE1 RNase inhibition limits hepatic CD95 expression and CD95mediated cell death in mice

A. Timeline of in vivo experiment 1. Eight mice were divided in two groups of 4 and repeatedly injected as indicated with either vehicle (group 1) or MKC-8866 (group 2). B. IHC staining for CD95 on liver tissue from the two groups of mice described in A. Left: quantification of CD95 staining. Mean \pm SEM of $n=4$ mice per group; * $p<0.05$ with Mann-Whitney test for comparison of the two groups. Right: representative IHC image for each group. D. IHC staining for cleaved caspase-3 on liver tissue from the two indicated groups of mice described in Fig S3A. Left: quantification of cleaved caspase3 staining. Mean \pm SEM of $n=10$ mice per group; ${ }^{* * *} p<0.0001$ Mann-Whitney test for comparison of the two indicated groups. Right: representative IHC image for each group. 
A

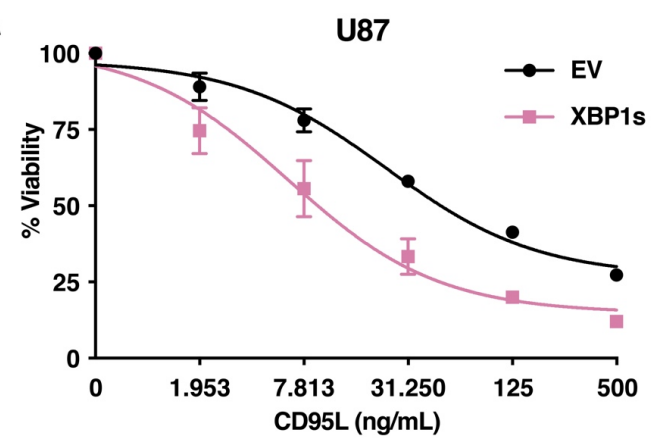

C
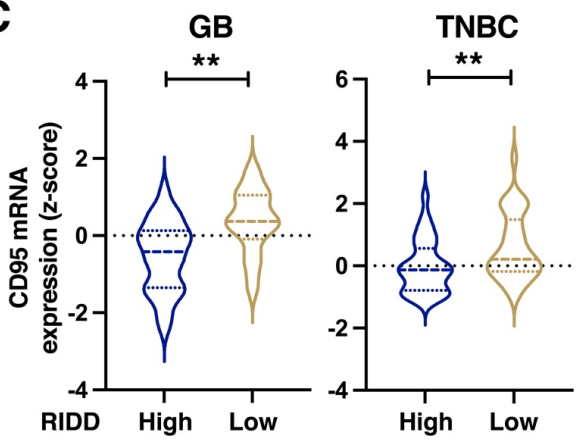

B
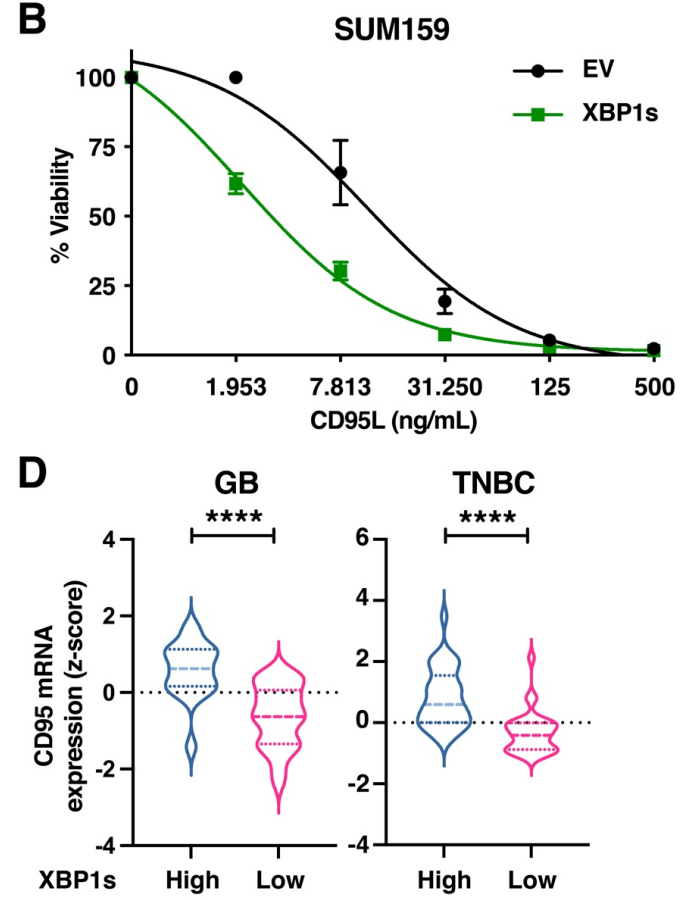

$E$

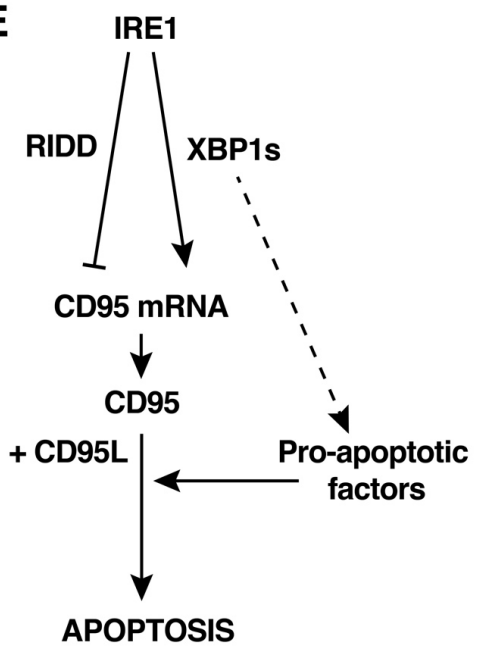

Figure 5 XBP1s promotes CD95L-induced death and the relative activation of each IRE1 branch correlates with CD95 expression in tumors

A. U87 cells were transfected with a plasmid coding for FLAG-XBP1s (XBP1s) or an empty vector (EV). 48 hours later, cells were treated with the indicated concentrations of CD95L for 48 hours. Viability was assessed using MTT assay and normalized to untreated cell values. Mean \pm SEM of 3 independent experiments. B. SUM159 cells were transfected with a plasmid coding for FLAG-XBP1s (XBP1s) or an empty vector (EV). 48 hours later, cells were treated with the indicated concentrations of CD95L for 48 hours. Viability was assessed using MTT assay and normalized to untreated cell values. Mean \pm SEM of 3 independent experiments. C, D. CD95 expression z-scores of 45 GB and 67 TNBC tumors were plotted according to the RIDD activity score (C) and according to the XBP1s activity score (D). The distribution of z-score is represented as violin plots. Statistical difference of expression between groups was calculated using Mann-Whitney tests and the $p$-value is indicated $\left({ }^{* *} p<0.01,{ }^{* * *} p\right.$ $\leq 0.0001) \mathrm{E}$. Scheme of the regulation of CD95 signal by IRE1 RNase based on the findings of the study. 